Curr Opin Rheumatol. 2011 September ; 23(5): 471-478. doi:10.1097/BOR.0b013e328349c2b1.

\title{
Inflammation in osteoarthritis
}

\author{
Mary B. Goldring and Miguel Otero \\ Research Division, The Hospital for Special Surgery, Weill Cornell Medical College, New York, \\ New York, USA
}

\begin{abstract}
Purpose of review-This review focuses on the novel stress-induced and proinflammatory mechanisms underlying the pathogenesis of osteoarthritis, with particular attention to the role of synovitis and the contributions of other joint tissues to cellular events that lead to the onset and progression of the disease and irreversible cartilage damage.

Recent findings-Studies during the past 2 years have uncovered novel pathways that, when activated, cause the normally quiescent articular chondrocytes to become activated and undergo a phenotypic shift, leading to the disruption of homeostasis and ultimately to the aberrant expression of proinflammatory and catabolic genes. Studies in animal models and retrieved human tissues indicate that proinflammatory factors may be produced by the chondrocytes themselves or by the synovium and other surrounding tissues, even in the absence of overt inflammation, and that multiple pathways converge on the upregulation of aggrecanases and collagenases, especially MMP-13. Particular attention has been paid to the contribution of synovitis in posttraumatic joint injury, such as meniscal tears, and the protective role of the pericellular matrix in mediating chondrocyte responses through receptors, such as discoidin domain receptor- 2 and syndecan- 4 . New findings about intracellular signals, including the transcription factors NF- $\kappa \mathrm{B}, \mathrm{C} / \mathrm{EBP} \beta, \mathrm{ETS}$, Runx 2 , and hypoxia-inducible factor- $2 a$, and their modulation by inflammatory cytokines, chemokines, adipokines, Toll-like receptor ligands, and receptor for advanced glycation endproducts, as well as $\mathrm{CpG}$ methylation and microRNAs, are reviewed.
\end{abstract}

Summary-Further work on mediators and pathways that are common across different models and occur in human osteoarthritis and that impact the osteoarthritis disease process at different stages of initiation and progression will inform us about new directions for targeted therapies.

\section{Keywords}

cartilage; inflammation; osteoarthritis; synovium

\section{Introduction}

Osteoarthritis has been considered a prototypical non-inflammatory arthropathy because neutrophils are absent in the synovial fluid, as are systemic manifestations of inflammation [1•]. The involvement of an inflammatory component, which is marked by symptoms such as joint pain, swelling and stiffness, is now well recognized. Inflammatory cytokines, chemokines, and other inflammatory mediators are produced by the synovium and chondrocytes, and can be measured in the synovial fluids of osteoarthritis patients (Fig. 1).

(C) 2011 Wolters Kluwer Health | Lippincott Williams \& Wilkins

Correspondence to Mary B. Goldring, PhD, The Hospital for Special Surgery, Caspary Research Building, 5th Floor, 535 East 70 th Street, New York, NY 10021, USA, Tel: +1 212774 7564; fax: +1 212 249 2373; goldringm@ @ss.edu.

Conflicts of interest

There are no conflicts of interest. 
Chondrocytes, the unique cellular component of adult human articular cartilage, maintain the matrix components under normal, low turnover conditions. In osteoarthritis, chondrocytes, but also the cells in the synovium and other joint tissues, become activated because of exposure to abnormal environmental insults, including high-magnitude mechanical stress, inflammatory cytokines, or altered amounts or organization of matrix proteins, including degradation products [2]. The activation of stress-induced and inflammation-induced signaling, transcriptional, and posttranscriptional events may cause phenotypic shift, apoptosis, and aberrant expression of inflammation-related genes, including catabolic genes [3]. These include nitric oxide synthase (NOS)-2, cyclooxygenase (COX)-2, and several matrix metalloproteinases (MMPs), including MMP-13, and a disintegrin and metalloproteinase (ADAM) with thrombospondin-1 domains (ADAMTS)-4 and 5. Although cartilage destruction is the hallmark of osteoarthritis and the degradation of type II collagen is the pivotal event that determines the irreversible progression of osteoarthritis disease, osteoarthritis is manifested in the 'whole joint' and thus we need to consider the synovium, meniscus, tendon, ligaments, and bone in the pathogenesis of the disease.

Risk factors for the development of osteoarthritis can be divided into two fundamental mechanisms related either to the adverse effects of trauma on an otherwise normal joint or of normal loading on a maligned joint. Either situation results in abnormal biomechanics. We have learned much from the studies of animal models of posttraumatic osteoarthritis, including transgenic and knockout mice subjected to surgical osteoarthritis [4], about the factors involved in cartilage degeneration and other joint pathologies. Genetic models with abnormal composition and structure of articular cartilage or other joint tissues may develop spontaneous or accelerated osteoarthritis because of altered biomechanics. Nevertheless, there are common mediators across these models that determine the initiation and progression of cartilage damage. Work during the past years has discovered the roles of a number of mediators that impact on the disease process and may inform us about new directions for targeted therapies [5].

\section{The role of synovitis in osteoarthritis}

Inflammation is a major factor associated with the risk of both progression of cartilage loss and signs and symptoms of disease, including joint pain, swelling, and stiffness, indicators of synovitis [6••]. Synovitis, involving infiltration of mononuclear cells into the synovial membrane and production of proinflammatory mediators, including interleukin $1 \beta$ (IL-1 $\beta$ ), tumor necrosis factor- $\alpha$ (TNF- $\alpha$ ), and chemokines, is common in early-stage and late-stage disease [6••]. MMP-1, MMP-3, and MMP-13 and cysteine cathepsins B and S, as well as IL-6, can be detected in osteoarthritis synovial fluid samples, although at significantly lower levels than in rheumatoid arthritis (RA) patients, reflecting the enhanced inflammatory processes in the RA joint [7]. The synovial effusions may be visualized in the joint by magnetic resonance imaging (MRI) or ultrasound [8]. The association between meniscal damage and synovial effusion has been noted by MRI. In patients with traumatic meniscal injury, but no radiographic evidence of osteoarthritis, the synovium retrieved during arthroscopic meniscectomy is frequently inflamed and increased inflammation scores are associated with increased pain and dysfunction and a unique chemokine profile [9••].

Epidemiologic studies have also established that there is a strong relationship between anterior cruciate ligament (ACL) disruption and risk for subsequent development of osteoarthritis. Following acute ACL injury, biomarkers of inflammation and collagen loss can be detected at higher levels in synovial fluid of the affected knee than in serum [10••], suggesting that low-grade synovitis reflects pre-clinical disease during early posttraumatic phase, which could impact on the long-term outcome [11]. The relevance of the synovial 
gene expression to global joint pathology is also supported by the findings of a high abundance synovial fluid proteome with distinct profiles in healthy individuals compared with early osteoarthritis patients undergoing arthroscopy after injury of the medial meniscus and late-stage patients undergoing total joint replacement [12].

\section{The role of stress and inflammatory factors in biomechanical responses in the osteoarthritis joint}

Chondrocytes can respond to direct biomechanical perturbation by upregulating synthetic activity or by increasing the production of inflammatory cytokines, which are also produced by other joint tissues. As mechanisms controlling relationships between joint injuries and biological events that lead to progressive joint degeneration cannot be evaluated over time in patients, both in-vitro and in-vivo models have been used to test hypotheses. The general consensus based on in-vitro mechanical loading experiments is that injurious static compression stimulates the depletion of proteoglycans and damage to the collagen network and decreases the synthesis of cartilage matrix proteins, whereas dynamic cyclic compression increases matrix synthetic activity. In response to traumatic injury, global gene expression is activated, resulting in increased expression of inflammatory mediators, cartilage-degrading proteinases, and stress-induced intracellular signals. Impact injury stimulates the release of reactive oxygen species (ROS) that induce chondrocyte death and activation of stress-induced kinases that upregulate MMP-13, ADAMTS-5, and TNF-a $[13,14]$. On the contrary, noninjurious cyclical loading of sufficient magnitude can inhibit IL-1-induced cartilage matrix degradation [15]. Thus, even in the absence of overt inflammation, chondrocytes may respond to mechanical stress by stimulating the expression and/or activities of inflammatory mediators or by inducing inhibitors that serve as feedback modulators.

\section{Role of cell-matrix interactions}

Chondrocytes have receptors for extracellular matrix (ECM) components, many of which are responsive to mechanical stimulation. Included among these receptors are several of the integrins, which serve as receptors for fibronectin and type II collagen fragments. Activation of these receptors can stimulate the production of matrix-degrading proteinases and inflammatory cytokines and chemokines, but whether these are initiating events or serve to feedback amplify matrix degradation has not been established. The importance of proteoglycan depletion in cartilage erosion was demonstrated in Adamts 5 knockout mice, which are protected against progression in the surgical osteoarthritis model. However, aggrecan depletion, by itself, does not drive cartilage erosion, as shown in recent studies in Mmp13 knockout mice, showing that MMP-13 deficiency inhibits osteoarthritis progression in the presence of aggrecan depletion [4].

In contrast to integrins, which bind collagen fragments, discoidin domain receptor 2 (DDR2) binds specifically to type II and X collagen fibrils, leading to the activation of its integral receptor tyrosine kinase. DDR2 is upregulated in osteoarthritis cartilage and induces specifically the expression of MMP-13 associated with cleavage of type II collagen. This mechanism was verified in type XI collagen-deficient (Cho/+) mice with accelerated osteoarthritis, which was attenuated, along with reduced MMP-13 expression, when they were crossed with mice deficient in DDR2 [16]. However, the type II collagen-containing network is normally not accessible because it is coated with proteoglycans and the pericellular collagen network is composed of type VI collagen microfibrils, not fibrillar collagen. Recent studies have shown that the serine proteinase, high temperature requirement A1 (HTRA1), which is increased in the articular cartilage of mouse models of osteoarthritis and human osteoarthritis cartilage, is responsible for degrading the pericellular 
matrix components, including matrilin 3, fibronectin, biglycan, fibromodulin, COMP, and collagen VI, thereby exposing DDR2 to activation by type II collagen in fibrillar form $[17 \bullet \bullet]$. Thus, DDR2 represents an additional receptor that becomes activated under biomechanical stress, leading to further disruption of the pericellular matrix and preferential activation of MMP-13.

Syndecan-4, a trans-membrane heparan sulfate proteoglycan that can interact with the ECM, is a positive effector of ADAMTS-5 activation by controlling the synthesis of the stromelysin, MMP-3 [18]. As syndecan-4 negatively regulates the function of osteopontin, which acts through integrin receptors, and osteopontin-deficient mice show accelerated development of osteoarthritis during aging and in an instability-induced model [19], the reduction of osteoarthritis in the syndecan-4 knockout mice may be because of unmasking of osteopontin activity.

Inflammation may also be driven by cartilage matrix molecules or degradation products through the activation of innate immune responses. Members of the small leucine-rich proteoglycan (SLRP) family such as fibromodulin and decorin may target the classic complement pathway and enhance or inhibit its activation [2]. COMP, however, is a potent activator of the alternative complement pathway and complexes of COMP and C3b may be found in osteoarthritis synovial fluids [20••].

\section{The role of cytokines and other inflammatory mediators}

Evidence from in-vivo and in-vitro studies indicates that synoviocytes, chondrocytes, and cells from other joint tissues can produce and/or respond to a number of cytokines and chemokines that may also be detected in osteoarthritis synovial fluid. Although the mechanism by which the inflammatory process is initiated in osteoarthritis is unclear, abnormal mechanical and oxidative stresses are probably involved. Chondrocytes in osteoarthritis cartilage, especially those in clonal clusters, express IL-1, IL-1 $\beta$-converting enzyme (caspase-1), and type 1 IL-1 receptor (IL-1RI). IL-1 is synthesized by chondrocytes at concentrations that are capable of inducing the expression of MMPs and other catabolic genes, and it colocalizes with TNF-a, MMP-1, MMP-3, MMP-8, and MMP-13, and type II collagen cleavage epitopes in regions of matrix depletion in osteoarthritis cartilage. The expression of MMPs is tightly regulated in chondrocytes under physiological conditions, wherein its expression pattern is restricted and the MMPs play essential roles in the lowturnover ECM remodeling.

The aggrecanases of the ADAMTS (a disintegrin and metalloproteinase with thrombospondin-1 domains) family of extracellular proteinases, particularly ADAMTS-5, are considered the major aggrecan-degrading enzymes involved in cartilage degradation in osteoarthritis. Several studies have shown a distinct regulation of ADAMTS-4 and ADAMTS-5 after inflammatory stimuli, depending upon the experimental conditions, the stimuli utilized, and the species analyzed. The mRNA expression of both enzymes is induced byTNF- $\alpha$ and oncostatin $M$ in both human and mouse chondrocytes [21,22], where as IL-1 appears to preferentially induce ADAMTS-4 [23].

In addition to inducing the synthesis of MMPs and other proteinases by chondrocytes, IL-1 and TNF- $\alpha$ increase the synthesis of prostaglandin $\mathrm{E}_{2}\left(\mathrm{PGE}_{2}\right)$ by stimulating the gene expression or activities of COX-2, microsomal PGE synthase-1 (mPGES-1), and soluble phospholipase A2 (sPLA2), and they upregulate the production of nitric oxide via inducible nitric oxide synthase (iNOS or NOS2). IL-1 $\beta$ and TNF- $\alpha$ may also induce other proinflammatory cytokines, such as IL-6, leukemia inhibitory factor, IL-17, and IL-18, and chemokines, including IL-8. Many of these factors synergize with one another in promoting chondrocyte catabolic responses. In addition, oncostatin $\mathrm{M}$ produces mild catabolic 
responses in chondrocytes through a gp130 receptor and JAK3, but synergizes strongly with IL-1 or TNF- $a$. IL-1 $\beta$ and TNF-a, alone or together, also suppress the expression of a number of genes associated with the differentiated chondrocyte phenotype, including aggrecan (ACAN) and type II collagen (COL2Al).

Both mechanical stress and inflammatory mediators induce an array of downstream signaling pathways, including theNF- $\mathrm{BB}$ and MAPK pathways, which are abnormally activated in osteoarthritis chondrocytes (for review see [3,24]). The NF- $\kappa$ B pathway is a central regulator of the inflammatory cytokine-induced catabolic actions in chondrocytes. Activation of canonical NF- $\mathrm{kB}$ (p65/p50) signaling is required for the chondrocytes to express MMPs, NOS2, COX2, and IL-1. Subsequently, the released ECM components are recognized by integrins and other cell-surface receptors, triggering inflammatory responses and further perpetuating the cartilage destruction. NF- $\kappa B$ also mediates the chondrocyte activation triggered by the released ECM products, such as fibronectin fragments, which induce the expression of inflammatory cytokines, MMPs, ADAMTSs, and chemokines.

In addition to NF- $\mathrm{BB}$, mechanical and inflammatory stimuli activate the stress-induced and mitogen-induced protein kinase (MAPK) pathways through ERK, c-Jun N-terminal kinase (JNK), and $\mathrm{p} 38$ kinase cascades in articular chondrocytes. The activation of these cascades coordinates the induction and activation of transcription factors, including members of the AP-1, ETS, and C/EBP families, that regulate the expression of genes involved in catabolic and inflammatory events. JNK-driven AP-1 activation, MEK/ERK-induced phosphorylation of ETS factors, and p38-mediated activation of C/EBP $\beta$ and RUNX2 participate in the induction of MMPs, including MMP-13 [25,26]. Such actions of particular kinases may be isoform-specific, as in the case of $\mathrm{p} 38 \gamma$, which actually suppresses MMP-13 production [27]. Another primary response factor for the regulation of cytokine-induced MMP-13 in chondrocytes is HIF-2a [28••], which is strongly induced by NF- $\kappa B$ signaling. For aggrecanases, transcriptional modulation involves RUNX2 for both ADAMTS-4 and ADAMTS-5 [29-31], whereas NF- $\kappa$ B [23] and HIF-2a consistently mediate ADAMTS-4 upregulation $[28 \bullet \bullet]$.

Recent studies have implicated synovial inflammation and secreted damage-associated molecular patterns (DAMPs), or alarmins, that act as ligands of Toll-like receptors (TLRs) or receptor for advanced glycation end-products (RAGEs), in the activation of downstream inflammatory and catabolic events in articular cartilage [5,6••] (Fig. 1). Chondrocytes and synovial cells can express TLRs, which are upregulated by inflammatory stimuli [32].

TLR-2 and TLR-4 levels are increased in osteoarthritis cartilage lesional areas, and activation of TLR-2 and TLR-4 by the ligands, peptidoglycan, and LPS, respectively, leads to increased expression of downstream inflammatory and catabolic genes, including MMP-3, MMP-13, and NOS2, through the cytosolic adaptor myeloid differentiation factor 88 (MyD88) and subsequent NF- $\kappa$ B signaling [33••,34]. The high-mobility group box (HMGB) protein 1 has been implicated in potentiating and contributing to osteoarthritis disease, acting on articular chondrocytes [35] or synoviocytes [36], by both responding to and enhancing inflammatory insults. Together with TLR ligands, HMGB1 acts as a cytokine-like signal of innate immunity to induce a hypertrophy-like phenotypic shift in osteoarthritis chondrocytes [33••]. The alarmins, S100A4, A8, A9, and A11, are also markers of destructive processes in the joint that signal through RAGE and TLRs to drive inflammation-associated matrix catabolism [37]. RAGE ligands can also increase ROS through upregulating cytokines and chemokines [38,39]. Zreiqat et al. [40] recently showed that homodimeric S100A8 or S100A9 can upregulate the expression of MMPs and ADAMTSs and mediate matrix degradation, but are expressed in early but not late experimental osteoarthritis. Findings in inflammatory arthritis models suggest that 
significant contributions of these ligands in osteoarthritis would require the presence of synovial activation [5].

Proinflammatory cytokines, prostaglandins, ROS, and nitric oxide may also cause oxidative stress and chondrocyte apoptosis by altering mitochondrial function [41,42]. Proteomics studies show that IL- $1 \beta$ and TNF- $a$ differentially regulate the proteins implicated in mitochondrial dysfunction [43], including the mitochondrial superoxide dismutase, SOD2, which may serve a protective role against ROS by scavenging free radicals, but may contribute to cell death upon long-term exposure. Mitochondrial functions are altered in osteoarthritis chondrocytes and synovial cells, wherein nitric oxide reduces the cell survival and inhibits the activity of the mitochondrial respiratory chain and ATP synthesis [44].

\section{Contributions of aging and obesity to inflammatory processes in osteoarthritis}

Although osteoarthritis is not inevitable as we grow old, aging is one of the most prominent risk factors for osteoarthritis onset and development. Chondrocytes in aging cartilage have impaired activity compared with normal chondrocytes, with evidence of a senescent secretory phenotype [45]. The increased accumulation and expression of both advanced glycation end-products (AGEs) and the AGE receptor, RAGE, that occur in aging chondrocytes are associated with dysregulated signaling pathways, altered synthetic activity, and increased synthesis of and enhanced sensitivity to cytokines and chemokines, which trigger the expression of MMPs and other inflammatory mediators $[39,45,46]$. The agingrelated loss of autophagy, a protective mechanism for normal chondrocytes that protects cells during stress responses, is associated with cell death and osteoarthritis development [47]. An earlier study [48] showed that decreased HIF-2a expression is associated with autophagy in osteoarthritis tissues and aging cartilage, and with elevated HIF-1a as a possible compensatory mechanism.

Obesity has become a major focus as an important risk factor for osteoarthritis development and progression, especially after the identification of white adipose tissue as an endocrine organ capable of secreting biologically active substances, the so-called adipokines.

Adipokines contribute to the low-grade inflammatory state of obese patients and may directly affect cartilage homeostasis. One source of adipokines in the joint is the infrapatellar fat pad, which contains nociceptive nerve fibers that could contribute to anterior knee pain [49]. Adipokines can also be produced by chondrocytes and act locally in promoting cartilage destruction in osteoarthritis. Higher leptin levels can be detected in osteoarthritis than in normal articular cartilage and a recent study [50] has shown that chondrocytes express a variety of adipokines whose expression is further induced by inflammatory stimuli. In addition, stimulation of articular chondrocytes with leptin, adiponectin, or resistin, alone or in combination with other inflammatory cytokines, induces and enhances the expression of cytokines, MMPs, and NOS2 [51,52]. The in-vitro studies performed to date strongly support the contributions of adipokines to inflammation and cartilage damage, but there is limited information from in-vivo models to determine whether they are protective or detrimental in vivo, or primary or secondary to the osteoarthritis development [53].

\section{Mechanisms involving epigenetics and microRNA}

In normal adult chondrocytes, as in all somatic cells, epigenetic mechanisms, involving DNA methylation at $\mathrm{CpG}$ sites, modifications of histone tails, and changes in chromatin structure, stabilize the phenotype. However, interactions with the environment may cause epigenetically induced changes in gene expression that are transmitted to daughter cells 
during many doublings. Previous studies have demonstrated epigenetic de-repression in association with loss of DNA methylation of genes such as MMP-3, MMP-9, MMP-13, ADAMTS4, IL-1 $\beta$, and leptin in chondrocytes with degradative phenotype in osteoarthritis cartilage, whereas DNA methylation does not play a role in differential expression of ADAMTS5 in osteoarthritis compared with normal chondrocytes. An inhibitor of NF- $\kappa B$, partly through modulation of DNA methyltransferase- 1 expression, can prevent cytokineinduced demethylation of a specific site in the IL- $1 \beta$ promoter associated with decreased expression of IL-1 $\beta$ [54]. A recent study [55] demonstrated that the genes encoding the suppressors of cytokine signaling (SOCS), SOCS2 and CIS-1, but not SOCS1 and SOCS3, are suppressed in osteoarthritis chondrocytes, but the CpG methylation status of the SOCS2 promoter is unchanged. Interestingly, loss of expression of osteogenic protein-1 (OP-1 or bone morphogenetic protein-7) in aging chondrocytes is associated with hypermethylation of the OP-1 promoter, and OP-1 treatment of chondrocytes downregulates the stress and inflammatory response genes [56].

How specific microRNAs could be influencing the cartilage homeostasis and disease mechanisms has also been receiving greater attention $[57 \bullet, 58,59,60 \bullet \bullet$. Of those reported so far, miR-140, miR-27a, miR-27b, and miR-146a all appear to attenuate ADAMTS and/or MMP expression $[60 \bullet \bullet, 61,62]$. For example, miR140, whose expression is reduced in osteoarthritis cartilage and suppressed by IL- $1 \beta$, downregulates the ADAMTS5 expression in normal cartilage. Furthermore, miR140 knockout mice are predisposed to age-related osteoarthritis-like changes and overexpression of miR140 in chondrocytes is protective against surgically induced osteoarthritis [60• ]. The synovial fluid concentrations of miR-16, miR-132, miR-146a, and miR-223 are reduced in osteoarthritis compared with healthy controls [63]. The importance of microRNAs in cartilage homeostasis is also supported by a study [64] showing that Sox 9 positively regulates COL2A1 in human chondrocytes via a miR-675-dependent mechanism.

\section{Conclusion}

Although much knowledge about inflammatory mediators in osteoarthritis has been gained in the last decade, further studies are needed to better define the mechanisms by which these factors tip the balance between homeostasis and activation to promote matrix destruction and cell death. In response to stress and inflammatory insults, osteoarthritis chondrocytes produce a variety of matrix-degrading enzymes, including metalloproteinases and aggrecanases. The expression of these degradative enzymes is dysregulated in osteoarthritis chondrocytes, and their increased and aberrant expression and activities are major contributors to cartilage degradation during osteoarthritis development and progression. As the signaling pathways involved in inflammatory and biomechanical stress are similar and these pathways may also induce and amplify the expression of cytokine and chemokine genes, it therefore remains controversial whether inflammatory mediators are primary or secondary regulators of cartilage damage and defective repair mechanisms in osteoarthritis. Although cytokines and their receptors, proteinase activities, and signaling kinases have been considered as targets for OA therapy, new knowledge about early stress-induced and inflammation-induced events involved at presymptomatic onset of the disease could provide valid biomarkers for diagnosis, as well as new therapeutic strategies.

\section{Acknowledgments}

M.B.G. research is supported in part by National Institutes of Health Grants R01-AG022021, R21-AR054887, and RC4 AR060546. M.O. has been supported by an Arthritis Foundation Postdoctoral Fellowship. 


\section{References and recommended reading}

Papers of particular interest, published within the annual period of review, have been highlighted as:

-of special interest

••of outstanding interest

Additional references related to this topic can also be found in the Current World Literature section in this issue (p. 501).

1. Vlad SC, Neogi T, Aliabadi P, et al. No association between markers of inflammation and osteoarthritis of the hands and knees. J Rheumatol. 2011 May 15. [Epub ahead of print]. This study reports the blood levels of 17 biomarkers of systemic inflammation are poorly associated with radiographic Kellgren and Lawrence scores in defined patient populations. However, the authors suggest that some markers (IL-6, adiponectin, LP-PLA2, and TNF-RII) warrant further investigation.

2. Heinegard D, Saxne T. The role of the cartilagematrix in osteoarthritis. Nat Rev Rheumatol. 2011; 7:50-56. [PubMed: 21119607]

3. Goldring MB, Otero M, Plumb DA, et al. Roles of inflammatory and anabolic cytokines in cartilage metabolism: signals and multiple effectors converge upon MMP-13 regulation in osteoarthritis. Eur Cell Mater. 2011; 21:202-220. [PubMed: 21351054]

4. Little CB, Fosang AJ. Is cartilage matrix breakdown an appropriate therapeutic target in osteoarthritis: insights from studies of aggrecan and collagen proteolysis? Curr Drug Targets. 2010; 11:561-575. [PubMed: 20199393]

5. Van den Berg WB. Osteoarthritis year 2010 in review: pathomechanisms. Osteoarthritis Cartilage. 2011; 19:338-341. [PubMed: 21324370]

6. Sellam J, Berenbaum F. The role of synovitis in pathophysiology and clinical symptoms of osteoarthritis. Nat Rev Rheumatol. 2010; 6:625-635. [PubMed: 20924410] This excellent review focuses on synovitis as amajor effector in the pathogenesis of osteoarthritis and cartilage loss.

7. Pozgan U, Caglic D, Rozman B, et al. Expression and activity profiling of selected cysteine cathepsins and matrix metalloproteinases in synovial fluids from patients with rheumatoid arthritis and osteoarthritis. Biol Chem. 2010; 391:571-579. [PubMed: 20180636]

8. Hayashi D, Roemer FW, Katur A, et al. Imaging of synovitis in osteoarthritis: current status and outlook. Semin Arthritis Rheum. 2011 Feb 2. [Epub ahead of print].

9. Scanzello CR, McKeon B, Swaim BH, et al. Synovial inflammation in patients undergoing arthroscopic meniscectomy: molecular characterization and relationship to symptoms. Arthritis Rheum. 2011; 63:391-400. [PubMed: 21279996] This study reports the importance of synovitis in determining whether patients with meniscal injury progress more rapidly to develop osteoarthritis.

10. Catterall JB, Stabler TV, Flannery CR, Kraus VB. Changes in serum and synovial fluid biomarkers after acute injury (NCT00332254). Arthritis Res Ther. 2010; 12:R229. [PubMed: 21194441] This study reports that of seven biomarkers measured during the first month after acute joint injury, significant correlations between the serum and synovial fluid concentrations were found for CtxI, NTx, osteocalcin, and MMP-3, very early treatment with agents to reduce inflammation or reduce collagen loss may have the potential to reduce the onset of future posttraumatic osteoarthritis.

11. Lotz MK, Kraus VB. New developments in osteoarthritis. Posttraumatic osteoarthritis: pathogenesis and pharmacological treatment options. Arthritis Res Ther. 2010; 12:211. [PubMed: 20602810]

12. Gobezie R, Kho A, Krastins B, et al. High abundance synovial fluid proteome: distinct profiles in health and osteoarthritis. Arthritis Res Ther. 2007; 9:R36. [PubMed: 17407561]

13. Ding L, Heying E, Nicholson N, et al. Mechanical impact induces cartilage degradation via mitogen activated protein kinases. Osteoarthritis Cartilage. 2010; 18:1509-1517. [PubMed: 20813194] 
14. Goodwin W, McCabe D, Sauter E, et al. Rotenone prevents impact-induced chondrocyte death. J Orthop Res. 2010; 28:1057-1063. [PubMed: 20108345]

15. Torzilli PA, Bhargava M, Park S, Chen CT. Mechanical load inhibits IL-1 induced matrix degradation in articular cartilage. Osteoarthritis Cartilage. 2010; 18:97-105. [PubMed: 19747586]

16. Xu L, Servais J, Polur I, et al. Attenuation of osteoarthritis progression by reduction of discoidin domain receptor 2 in mice. Arthritis Rheum. 2010; 62:2736-2744. [PubMed: 20518074]

17. Polur I, Lee PL, Servais JM, et al. Role of HTRA1, a serine protease, in the progression of articular cartilage degeneration. Histol Histopathol. 2010; 25:599-608. [PubMed: 20238298] This study highlights the importance of the pericellular matrix in protection against chondrocyte-mediated cartilage loss.

18. Echtermeyer F, Bertrand J, Dreier R, et al. Syndecan-4 regulates ADAMTS-5 activation and cartilage breakdown in osteoarthritis. Nat Med. 2009; 15:1072-1076. [PubMed: 19684582]

19. Matsui Y, Iwasaki N, Kon S, et al. Accelerated development of aging-associated and instabilityinduced osteoarthritis in osteopontin-deficient mice. Arthritis Rheum. 2009; 60:2362-2371. [PubMed: 19644889]

20. Happonen KE, Saxne T, Aspberg A, et al. Regulation of complement by cartilage oligomeric matrix protein allows for a novel molecular diagnostic principle in rheumatoid arthritis. Arthritis Rheum. 2010; 62:3574-3583. [PubMed: 20737467] This study reports that COMP is a potent activator of the alternative complement pathway and that complexes of COMP and C3b may be found in osteoarthritis synovial fluids.

21. Song RH, Tortorella MD, Malfait AM, et al. Aggrecan degradation in human articular cartilage explants is mediated by both ADAMTS-4 and ADAMTS-5. Arthritis Rheum. 2007; 56:575-585. [PubMed: 17265492]

22. Rogerson FM, Chung YM, Deutscher ME, et al. Cytokine-induced increases in ADAMTS-4 messenger RNA expression do not lead to increased aggrecanase activity in ADAMTS-5-deficient mice. Arthritis Rheum. 2010; 62:3365-3373. [PubMed: 20662062]

23. Gabay O, Sanchez C, Salvat C, et al. Stigmasterol: a phytosterol with potential antiosteoarthritic properties. Osteoarthritis Cartilage. 2010; 18:106-116. [PubMed: 19786147]

24. Marcu KB, Otero M, Olivotto E, et al. NF-kappaB signaling: multiple angles to target OA. Curr Drug Targets. 2010; 11:599-613. [PubMed: 20199390]

25. Liu FC, Hung LF, Wu WL, et al. Chondroprotective effects and mechanisms of resveratrol in advanced glycation end products-stimulated chondrocytes. Arthritis Res Ther. 2010; 12:R167. [PubMed: 20825639]

26. Nishitani K, Ito H, Hiramitsu T, et al. PGE2 inhibits MMP expression by suppressing MKK4-JNK MAP kinase-c-JUN pathway via EP4 in human articular chondrocytes. J Cell Biochem. 2010; 109:425-433. [PubMed: 19998410]

27. Long DL, Loeser RF. p38gamma mitogen-activated protein kinase suppresses chondrocyte production of MMP-13 in response to catabolic stimulation. Osteoarthritis Cartilage. 2010; 18:1203-1210. [PubMed: 20633667]

28. Yang S, Kim J, Ryu JH, et al. Hypoxia-inducible factor-2alpha is a catabolic regulator of osteoarthritic cartilage destruction. Nat Med. 2010; 16:687-693. [PubMed: 20495569] This study reports HIF-2 $a$ as a novel regulator of cartilage destruction in osteoarthritis that is induced by inflammatory cytokines.

29. Tetsunaga T, Nishida K, Furumatsu T, et al. Regulation of mechanical stress-induced MMP-13 and ADAMTS-5 expression by RUNX-2 transcriptional factor in SW1353 chondrocyte-like cells. Osteoarthritis Cartilage. 2011; 19:222-232. [PubMed: 21094261]

30. Thirunavukkarasu K, Pei Y, Moore TL, et al. Regulation of the human ADAMTS-4 promoter by transcription factors and cytokines. Biochem Biophys Res Commun. 2006; 345:197-204. [PubMed: 16677612]

31. Thirunavukkarasu K, Pei Y, Wei T. Characterization of the human ADAMTS-5 (aggrecanase-2) gene promoter. Mol Biol Rep. 2007; 34:225-231. [PubMed: 17211519]

32. Geurts J, van den Brand BT, Wolf A, et al. Toll-like receptor 4 signalling is specifically TGF-betaactivated kinase 1 independent in synovial fibroblasts. Rheumatology (Oxford). 2011; 50:12161225. [PubMed: 21335610] 
33. Liu-Bryan R, Terkeltaub R. Chondrocyte innate immune myeloid differentiation factor 88dependent signaling drives procatabolic effects of the endogenous Toll-like receptor 2/Toll-like receptor 4 ligands low molecular weight hyaluronan and high mobility group box chromosomal protein 1 in mice. Arthritis Rheum. 2010; 62:2004-2012. [PubMed: 20506365] This report shows that HMGB1, together with TLR-2 and 4 ligands and MyD88, acts as a cytokine-like signal of innate immunity to induce a hypertrophy-like phenotypic shift in osteoarthritis chondrocytes.

34. Campo GM, Avenoso A, Campo S, et al. Molecular size hyaluronan differently modulates toll-like receptor-4 in LPS-induced inflammation in mouse chondrocytes. Biochimie. 2010; 92:204-215. [PubMed: 19879319]

35. Heinola T, Kouri VP, Clarijs P, et al. High mobility group box-1 (HMGB-1) in osteoarthritic cartilage. Clin Exp Rheumatol. 2010; 28:511-518. [PubMed: 20659415]

36. Garcia-Arnandis I, Guillen MI, Gomar F, et al. High mobility group box 1 potentiates the proinflammatory effects of interleukin-1beta in osteoarthritic synoviocytes. Arthritis Res Ther. 2010; 12:R165. [PubMed: 20799933]

37. Miranda KJ, Loeser RF, Yammani RR. Sumoylation and nuclear translocation of S100A4 regulate IL-1beta-mediated production of matrix metalloproteinase-13. J Biol Chem. 2010; 285:3151731524. [PubMed: 20685652]

38. Kapoor M, Martel-Pelletier J, Lajeunesse D, et al. Role of proinflammatory cytokines in the pathophysiology of osteoarthritis. Nat Rev Rheumatol. 2011; 7:33-42. [PubMed: 21119608]

39. Rasheed Z, Akhtar N, Haqqi TM. Advanced glycation end products induce the expression of interleukin- 6 and interleukin- 8 by receptor for advanced glycation end product-mediated activation of mitogen-activated protein kinases and nuclear factor-\{kappa $\} \mathrm{B}$ in human osteoarthritis chondrocytes. Rheumatology (Oxford). 2011; 50:838-851. [PubMed: 21172926]

40. Zreiqat H, Belluoccio D, Smith MM, et al. S100A8 and S100A9 in experimental osteoarthritis. Arthritis Res Ther. 2010; 12:R16. [PubMed: 20105291]

41. Blanco FJ, Rego I, Ruiz-Romero C. The role of mitochondria in osteoarthritis. Nat Rev Rheumatol. 2011; 7:161-169. [PubMed: 21200395]

42. Kim J, Xu M, Xo R, et al. Mitochondrial DNA damage is involved in apoptosis caused by proinflammatory cytokines in human OA chondrocytes. Osteoarthritis Cartilage. 2010; 18:424-432. [PubMed: 19822235]

43. Cillero-Pastor B, Ruiz-Romero C, Carames B, et al. Proteomic analysis by two-dimensional electrophoresis to identify the normal human chondrocyte proteome stimulated by tumor necrosis factor alpha and interleukin-1beta. Arthritis Rheum. 2010; 62:802-814. [PubMed: 20131227]

44. Cillero-Pastor B, Martin MA, Arenas J, et al. Effect of nitric oxide on mitochondrial activity of human synovial cells. BMC Musculoskelet Disord. 2011; 12:42. [PubMed: 21303534]

45. Shane Anderson A, Loeser RF. Why is osteoarthritis an age-related disease? Best Pract Res Clin Rheumatol. 2010; 24:15-26. [PubMed: 20129196]

46. Loeser RF. Age-related changes in the musculoskeletal system and the development of osteoarthritis. Clin Geriatr Med. 2010; 26:371-386. [PubMed: 20699160]

47. Carames B, Taniguchi N, Otsuki S, et al. Autophagy is a protective mechanism in normal cartilage, and its aging-related loss is linked with cell death and osteoarthritis. Arthritis Rheum. 2010; 62:791-801. [PubMed: 20187128]

48. Bohensky J, Terkhorn SP, Freeman TA, et al. Regulation of autophagy in human and murine cartilage: hypoxia-inducible factor 2 suppresses chondrocyte autophagy. Arthritis Rheum. 2009; 60:1406-1415. [PubMed: 19404942]

49. Clockaerts S, Bastiaansen-Jenniskens YM, Runhaar J, et al. The infrapatellar fat pad should be considered as an active osteoarthritic joint tissue: a narrative review. Osteoarthritis Cartilage. 2010; 18:876-882. [PubMed: 20417297]

50. Conde J, Gomez R, Bianco G, et al. Expanding the adipokine network in cartilage: identification and regulation of novel factors in human and murine chondrocytes. Ann Rheum Dis. 2011; 70:551-559. [PubMed: 21216818]

51. Kang EH, Lee YJ, Kim TK, et al. Adiponectin is a potential catabolic mediator in osteoarthritis cartilage. Arthritis Res Ther. 2010; 12:R231. [PubMed: 21194467] 
52. Zhang Z, Xing X, Hensley G, et al. Resistin induces expression of proinflammatory cytokines and chemokines in human articular chondrocytes via transcription and messenger RNA stabilization. Arthritis Rheum. 2010; 62:1993-2003. [PubMed: 20506172]

53. Griffin TM, Fermor B, Huebner JL, et al. Diet-induced obesity differentially regulates behavioral, biomechanical, and molecular risk factors for osteoarthritis in mice. Arthritis Res Ther. 2010; 12:R130. [PubMed: 20604941]

54. Imagawa K, de Andres MC, Hashimoto K, et al. The epigenetic effect of glucosamine and a nuclear factor-kappa B (NF-kB) inhibitor on primary human chondrocytes: implications for osteoarthritis. Biochem Biophys Res Commun. 2011; 405:362-367. [PubMed: 21219853]

55. De Andres MC, Imagawa K, Hashimoto K, et al. Suppressors of cytokine signalling (SOCS) are reduced in osteoarthritis. Biochem Biophys Res Commun. 2011; 407:54-59. [PubMed: 21352802]

56. Chubinskaya S, Otten L, Soeder S, et al. Regulation of chondrocyte gene expression by osteogenic protein-1. Arthritis Res Ther. 2011; 13:R55. [PubMed: 21447156]

57. Araldi E, Schipani E. MicroRNA-140 and the silencing of osteoarthritis. Genes Dev. 2010; 24:1075-1080. [PubMed: 20516192] This review article summarizes many recent findings that point to microRNAs as homeostatic regulators of chondrocyte function with particular attention to the work by Miyaki et al. [60••].

58. Alcaraz MJ, Megias J, Garcia-Arnandis I, et al. New molecular targets for the treatment of osteoarthritis. Biochem Pharmacol. 2010; 80:13-21. [PubMed: 20206140]

59. Kim D, Song J, Jin EJ. MicroRNA-221 regulates chondrogenic differentiation through promoting proteosomal degradation of slug by targeting Mdm2. J Biol Chem. 2010; 285:26900-26907. [PubMed: 20576614]

60. Miyaki S, Sato T, Inoue A, et al. MicroRNA-140 plays dual roles in both cartilage development and homeostasis. Genes Dev. 2010; 24:1173-1185. [PubMed: 20466812] This is a comprehensive report on the critical role that microRNA-140 plays in the development and maintenance of cartilage and how its decrease in osteoarthritis my contribute to the loss of homeostasis.

61. Li X, Gibson G, Kim JS, et al. MicroRNA-146a is linked to pain-related pathophysiology of osteoarthritis. Gene. 2011; 480:34-41. [PubMed: 21397669]

62. Akhtar N, Rasheed Z, Ramamurthy S, et al. MicroRNA-27b regulates the expression of matrix metalloproteinase 13 in human osteoarthritis chondrocytes. Arthritis Rheum. 2010; 62:1361-1371. [PubMed: 20131257]

63. Murata K, Yoshitomi H, Tanida S, et al. Plasma and synovial fluid microRNAs as potential biomarkers of rheumatoid arthritis and osteoarthritis. Arthritis Res Ther. 2010; 12:R86. [PubMed: 20470394]

64. Dudek KA, Lafont JE, Martinez-Sanchez A, Murphy CL. Type II collagen expression is regulated by tissue-specific miR-675 in human articular chondrocytes. J Biol Chem. 2010; 285:2438124387. [PubMed: 20529846] 


\section{Key points}

- Osteoarthritis is a whole joint disorder, affecting all joint tissues that communicate at the cellular level by releasing and responding to inflammatory mediators.

- Synovial inflammation acts as a trigger for several symptoms of osteoarthritis via release of soluble factors that, while increasing and perpetuating cartilage damage, hold the potential to be used as biomarkers.

- Inflammation may act as a contributing factor in perpetuating cartilage degradation by promoting destruction and impairing the ability of repair.

- A wide array of injurious stimuli activate stress/inflammatory responses that lead to a phenotypic shift in osteoarthritis chondrocytes, which show features of hypertrophy and imbalanced homeostasis, with increased synthetic activities in which catabolic responses overcome the attempt of repair. 


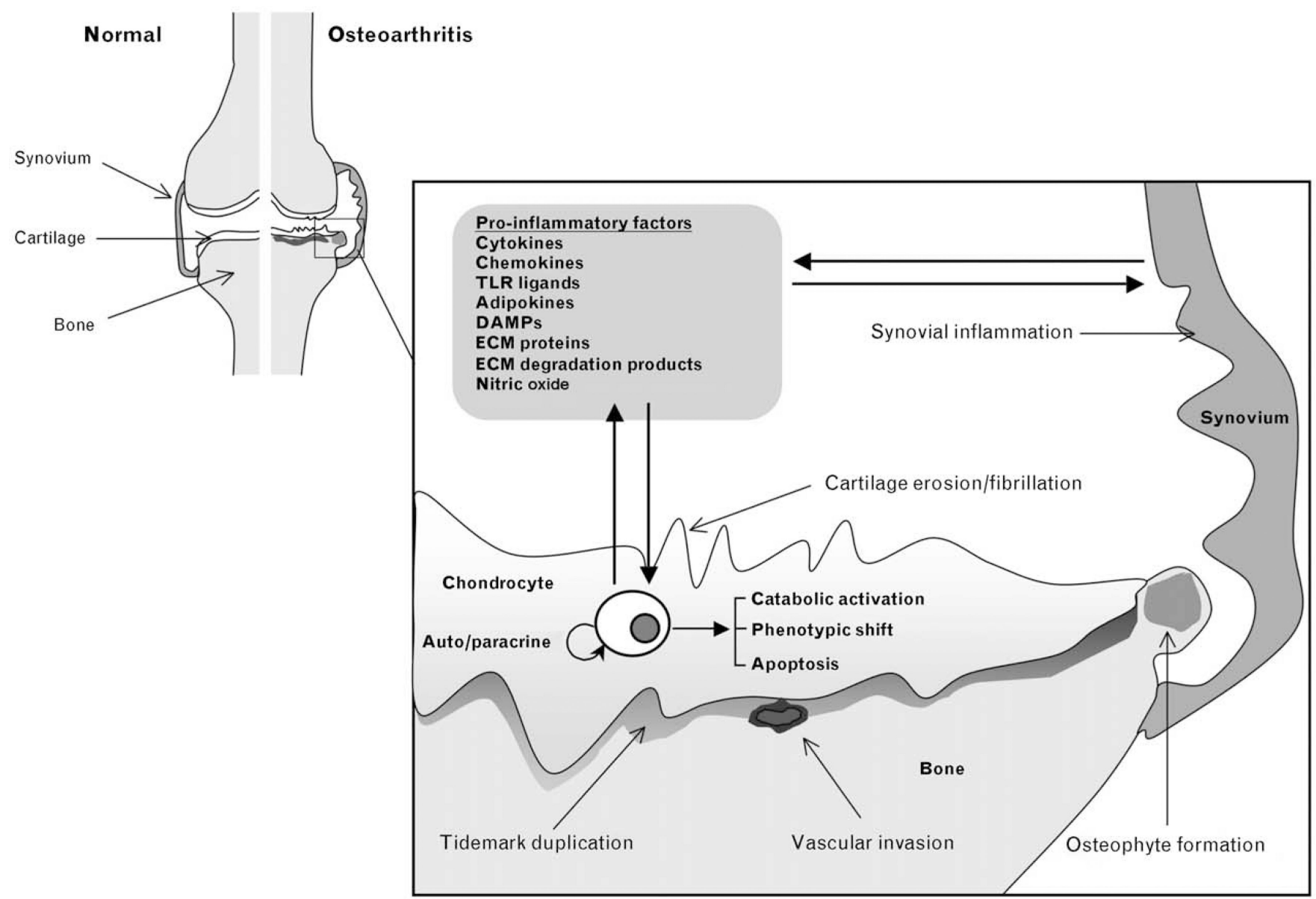

Figure 1. Key mediators involved in proinflammatory events in osteoarthritis

In response to alterations in the joint environment, inflammation-induced and stress-induced signaling pathways may be activated in the synovium and cartilage resulting in the production of cytokines, chemokines, adipokines, Toll-like receptor (TLR) ligands, and other inflammatory mediators such as nitric oxide. This may occur also in response to alterations in the pericellular matrix or the release of damage-associated molecular patterns, which are TLR and RAGE ligands. The consequent upregulation of cartilage-degrading proteinases by extracellular matrix (ECM) proteins or ECM degradation products can further promote catabolic activation, phenotypic shift, and apoptosis. 